\title{
微痕观察初步确认灵井许昌人遗址旧石器时代骨制工具
}

\author{
李占扬 ${ }^{(1)}$, 沈辰 ${ }^{2(3) *}$ \\ (1) 河南省文物考古研究所, 郑州 450000; \\ (2) 中国科学院人类演化与科技考古联合实验室, 北京 100044 ; \\ (3) The Royal Ontario Museum of Canada, Toronto M5S2C6, Canada; \\ (4) 山东大学东方考古研究中心, 济南 250100 \\ *联系人, E-mail: chens@ rom.on.ca
}

2009-07-06 收稿, 2009-10-14 接受

国家自然科学基金(批准号: 40872115, 40602006)、中国科学院-国家外国专家局创新团队国际合作伙伴计划、高等学校学科创新引智计划(编 号: 111-2-09)和 Royal Ontario Museum Research Grant 资助项目

摘要 2007 2008 年河南灵井许昌人遗址发现距今 10 8 万年前的古人类头骨化石，与头骨 化石同层出土有大量哺乳动物骨骼化石. 部分动物骨骼中可能存在经人工改造的骨制品. 通过对遗址出土的部分骨骼化石进行微痕观察并与实验标本对比分析，能辨认出灵井骨制 品上确有锥钻、穿刺、刮削等使用痕迹以及疑似捆绑的微痕. 研究确认中国北方晚更新世早 期的旧石器时代遗址中，存在着有意识加工制作和使用过的骨制工具. 这一发现也证实了骨

关键词

河南许昌人遗址

模拟实验

微痕分析

骨制品 制工具的使用与石器一样, 是古人类技术发展和行为文化的一个重要方面.

河南灵井许昌人遗址是中国近年来旧石器时代 考古的重大发现之一. 图 1 是遗址位置图. 2005 2009 年间，河南省文物考古研究所对位于河南省许昌市 的该遗址进行连续调查与发掘, 获得旧石器时代的 石制品和动物化石等 3 万余件. 更为重要的是在 2007 和 2008 两年度的考古发掘中, 出土了一批古人 类头骨化石断块，现场观察属同一个体. 目前，对该 遗址文化遗物包括石器和动物化石的研究已发表了 一批成果 ${ }^{[1 \sim 3]}$.

通过对该遗址出土的哺乳动物化石的研究, 李 占扬和董为认为灵井动物群的时代应与许家窑动物 群的时代相似, 为晚更新世早期 ${ }^{[4]}$. 根据光释光测年, 初步认为出土古人类化石的文化层的年代为距今 10 8 万年 ${ }^{[5]}$.

灵井许昌人遗址的埋藏类型以典型的湖相沉积 为主. 遗物保存状况良好, 动物骨骼基本没有风化.
经过初步鉴定，出土的动物骨骼中有 103 件被认定是 经人工加工过的骨制品 ${ }^{[6]}$. 按传统类型学分析, 根据 加工修理部位的不同可将器型分为刮削器、尖状器、 尖刃两用器和雕刻器 4 类, 其中以刮削器最多. 在此 基础上, 本文应用微痕分析方法对出土的部分骨制 品进行初步观察. 这是国内运用微痕分析技术对旧 石器时代遗址出土的骨制品的创新性研究.

\section{1 研究目的与方法}

考古遗存中出土的动物化石是常见的骨质遗物, 无论是完整的还是破碎的, 都是旧石器时代考古资 料的重要组成部分. 而骨制品是古人类有意识地对 动物骨骼进行改造后的产品. 这种人工改造包括敲 骨吸髓、割骨剔肉、刮骨去脂等生存行为, 更重要的 是加工制作各种类型的工具并加以使用. 不是所有 的骨制品都被用作史前工具, 只有那些能被确认被 


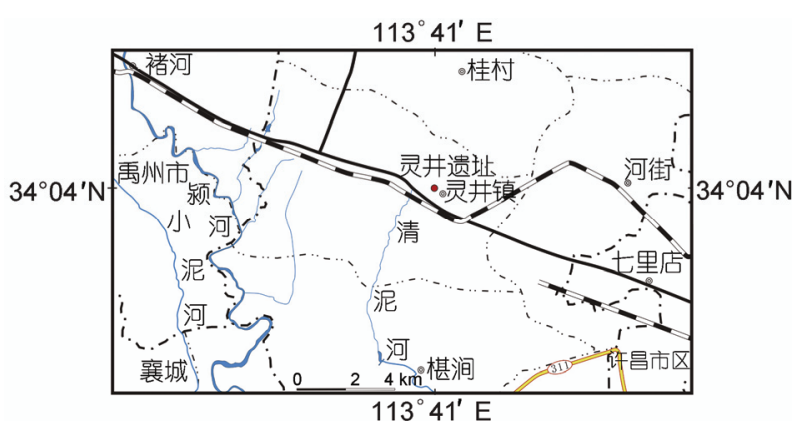

图 1 河南灵井许昌人遗址位置图

有意识地加工成工具并经过使用的部分骨制品才被 称为骨制工具或骨器. 因此, 我们对灵井遗址骨制品 的研究首先需要解决的一个问题是: 距今 10 8 万年 的许昌人是否是有意识地制作和使用骨制工具?

考古资料及相应研究显示, 从旧石器时代早期 开始, 人类逐渐开始有意识地利用动物骨骼 ${ }^{[7]}$. 过去 对骨制品人工改造性质的认定往往依赖于骨制品的 形状, 并由其形状推测其制作方法和使用功能 ${ }^{[8]}$. 但 由于埋藏环境、自然风化、动物或人类踩踏等诸多因 素都可能对骨骼破裂和表面改造产生影响, 因此仅 用形态特征并不能准确说明骨制品的形成与使用过 程. 为了尽可能全面客观地认识骨骼破裂的原因, 区 分出自然和人工因素, 进而提炼人类利用动物骨骼 的行为信息, 我们可以通过观察动物骨骼上的各种 痕迹, 在对比实验标本的基础上, 分析遗址中骨骼标 本是否存在有意识加工及使用的痕迹. 特别是通过 借助各类显微镜, 对骨制品的工作刃缘或表面进行 细微的观察, 分析其在制作和使用中产生的各类痕 迹, 包括擦痕、破碎疤痕等的组合、分布、方向性以 及经过使用后产生的磨圆和光泽等, 以确认其制作 方法和使用功能. 同时尽可能明确不同因素下形成 多种类型痕迹的标准，探讨不同背景下形成的痕迹 有无规律. 上述研究手段即是微痕分析方法 ${ }^{[9]}$.

微痕分析方法最早由前苏联考古学家西蒙诺夫 创立, 20 世纪末在欧美地区广泛应用于石器功能研 究 ${ }^{[10 ~ 14]}$. 微痕分析在石器研究中的成功推动了它在 骨器研究领域的应用 ${ }^{[15 ~ 21]}$. 近 20 年来, 国外对骨制 品的微痕研究发展迅速, 有不少成功实验和研究个 案的成果相继发表 ${ }^{[22 ~ 24]}$. 国内虽已经做过较多相应 的基础工作, 如对动物骨骼破碎原因和动物骨骼表 面痕迹的研究已开始并有所突破 ${ }^{[25,26]}$, 但利用微痕
方法对骨制品人工性质的研究还处于空白阶段. 基 于以上因素, 我们目前的研究目的有两个: 一个是在 已有工作的基础上，借鉴石制品已确定的微痕分析 标准, 观察、分析、判断灵井许昌人遗址骨制品上使 用痕迹的特征及成因; 另一个是结合遗址和遗物的 具体特点, 对今后骨器微痕研究方法和研究设计作 进一步探讨.

\section{2 实验骨制品的微痕观察}

微痕分析的基础是实验 ${ }^{[27]}$. 骨制品微痕的鉴别 和认定需要通过与对相似工具和加工材料的实验操 作所产生的痕迹进行比照. 对骨器使用功能的阐述 也需要经得起实验数据的检验.

针对是否能从动物骨骼上区分出人工改造和使 用的痕迹, 我们的实验工作主要分为两类：一类是以 石块或钢刀为工具作用于动物骨骼, 包括砍砸、刮肉 和脚踩的动作, 主要观察动物骨骼因人类有意识作 用于其上的行为所形成的痕迹的特征; 另一类则是 以动物骨骼为工具或动作主体, 作用于其他物体上, 主要观察骨制品因使用所产生的痕迹.

\section{1 实验过程}

用 6 件动物骨骼做实验. 实验对象为羊的肩胛骨 和肱骨各 3 件(编号分别为 EXP001 EXP006), 均为 新鲜骨头, 但经过约 $3 \mathrm{~d}$ 的冷藏. 实验时, 这些骨骼 仍含有一定水分, 油脂比较丰富, 并附有薄层羊肉. 第一部分 (改造) 实验过程如下: 在两件肩胛骨 (EXP001 和 EXP002)上用刀不锋利的中小型砾石石 块刮肉, 目的是将附着在骨骼上的肉基本剔除掉, 以 留下刮削痕; 用中小型石块砍砸两件肱骨(EXP003 和 EXP004), 石块刃缘与肱骨长轴垂直, 目的是通过 砍砸以获取能制作工具的骨坏，同时观察在肱骨上 留下的砍砸痕; 将 1 件肱骨(EXP005)和 1 件肩胛骨 (EXP006)放在细砂土中磨滚, 间或用脚踩磨, 目的是 观察比较非人工意识的改造骨骼表面的痕迹.

在第二部分(使用)实验过程中, 选择其中 2 件标 本作为工具使用. 首先用标本 EXP002 的尖端钻皮革, 反复旋钻, 操作持续 $12 \mathrm{~min}$; 随后用此尖端的两个侧 面磨擦皮革, 左右反复运动, 连续操作 $10 \mathrm{~min}$. 同时, 用标本 EXP003 尖端的一个侧缘磨擦皮革, 左右反复 运动, 动作较快, 持续操作 $12 \mathrm{~min}$. 


\section{2 实验标本微痕观察}

在实验标本放大 30 倍和 40 倍的情况下观察人工 改造的微痕. EXP001 主要用于骨骼表面上刮肉整骨 的实验, 观察到工作面上有成组的纵向、横向和斜向 平行条痕(相对于肩胛骨长轴而言)。标本 EXP002 在 放大 14 倍和 25 倍时, 能够观察到由刮肉产生的光泽 和由砍砸产生的砍痕和裂疮(图 2(a)). 标本 EXP003 在显微镜下放大 14 倍后可以观察到敲骨吸髓砸痕周 缘的系列中小型破裂疮(图 2(b)). EXP002 钻皮革的尖 端周缘的脊上在刮磨实验中留下了明显的光泽和较 为重度的磨圆, 产生的痕迹与 EXP003 磨擦皮革实验 的结果基本相同. 但相比之下 EXP003 上的痕迹不甚 鲜明.

通过观察实验标本的微痕，我们认识到，人类制 作和使用骨制品所留下的痕迹是可以通过微痕分析 与非人工或自然因素产生的痕迹区别开来的. 近年 来欧美学者的骨制品微痕实验工作同样证实了这一 点 ${ }^{[22 ~ 24]}$. 这些实验数据为我们鉴别灵井许昌人遗址 中骨制品制作加工痕迹和使用痕迹提供了可靠的 参考.

\section{3 灵井遗址骨制品微痕观察及其结果}

\section{1 出土标本的微痕描述}

本文所分析的出土标本为灵井许昌人遗址 2005 年出土的部分骨制品(图 3), 2006 年 4 月在河南省文 物考古研究所对其进行初步微痕观察. 使用的是国 产北京福凯体式显微镜, 放大倍数最大到 45 倍. 由
于工作时间有限，我们从初步认定的骨制品中随机 抽样, 在不同出土单位中具有明显加工修理特征并 且在原始报告中已做器型分类的标本中挑出 11 件样 本. 在低倍显微镜下观察, 发现这些样本有明显的痕 迹，其特征证实了标本的一些后埋藏、人工制作和使 用情况. 下面对骨器标本的描述是以骨骼外表朝上 面对观察者, 以此确定左右侧方位; 骨骼上下的位置 以绘图方向为准(图 3).

(i) 标本 5L442. 该标本尖端有系列连续分布 的中型片疮, 尾端形态为羽状和卷边状, 而且严重磨 圆并产生明亮光泽及纵横交错的条痕, 使用痕迹明 显. 背面中部有光泽, 可能是由自然风化产生的. 左 边刃出现有中度磨圆, 与直接使用有关. 右边刃的磨圆 和擦痕不明显, 没有明显的光泽和片疤，基本保留自然 面. 上部的大型阶梯状疤可能是砍砸造成的. 综上, 我 们认为该标本具有两种功能, 即向下切人的运动和单 一的表面接触, 使用区域集中在左侧边缘和尖端.

(ii) 标本 5L219. 该标本背面有风化光泽. 右 侧中部刃缘分布着一组大型羽状片疤，其边缘有系 列密集的细碎小疤. 该边缘相应的骨骼内面具有使 用光泽和一定程度的磨圆. 若这些痕迹由使用造成, 那么这件标本应该是用于刮削软性物质的。

(iii) 标本 5L217. 该标本最典型的微痕特征是 表面存在非常明显的多组交错条痕，同时两个尖端 都具有明显的使用痕迹. 其上部尖端有重度磨圆, 出 现数个破裂片疤以及由尖端向骨体内部延伸的方向 性的条痕. 上述特征说明, 这个区域可能具有雕刻功 能(图 4). 而下部尖端有折断型片疤, 另有若干由旋转
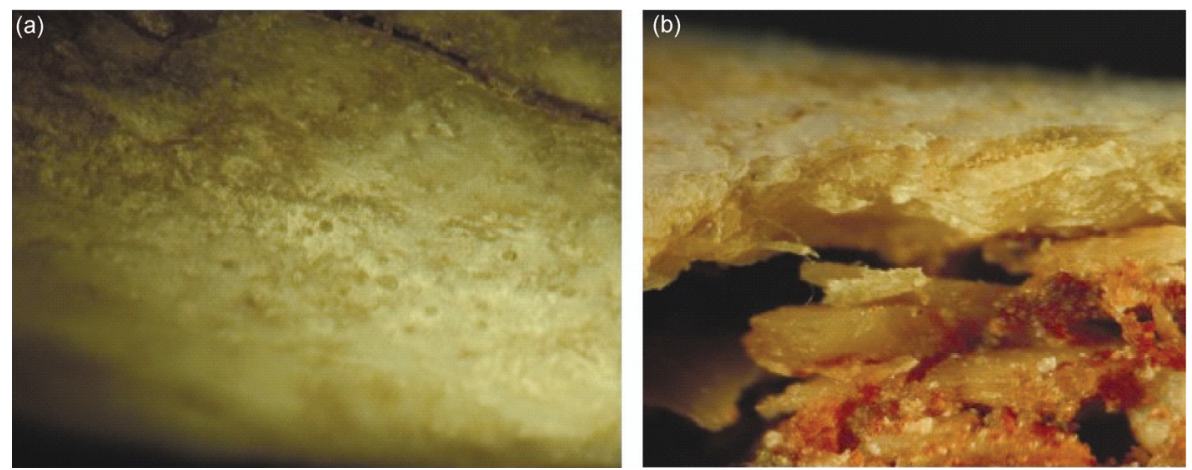

图 2 骨制品实验标本的微痕观察

(a) 标本 EXP002 骨骼表面通过有意识地刮骨改造之后产生的光泽和砄痕; (b) 标本 EXP003 在羊肱骨上敲骨砍砸后周缘产生的一系列中小型 破裂疮 

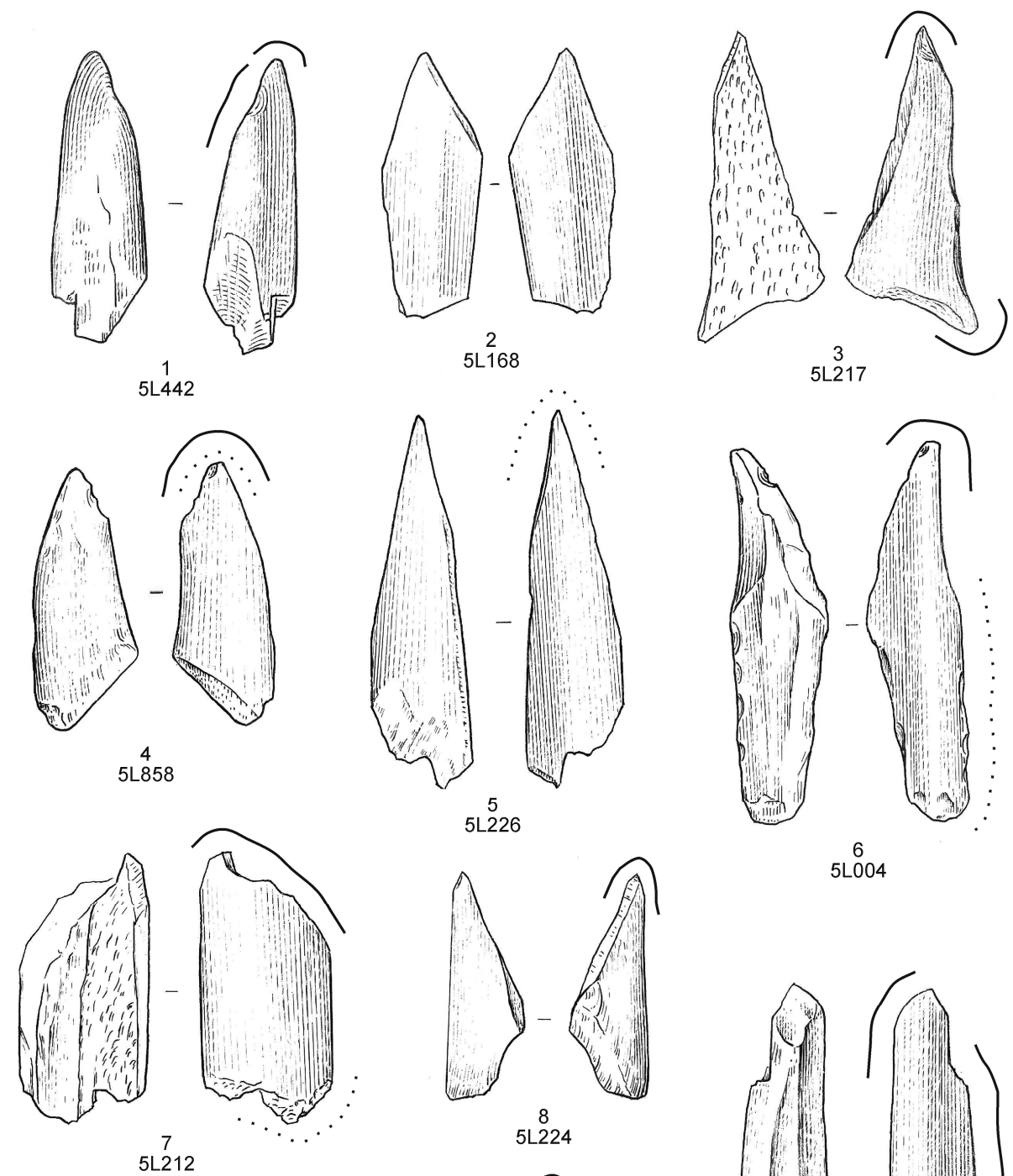

$\stackrel{3}{5} 217$
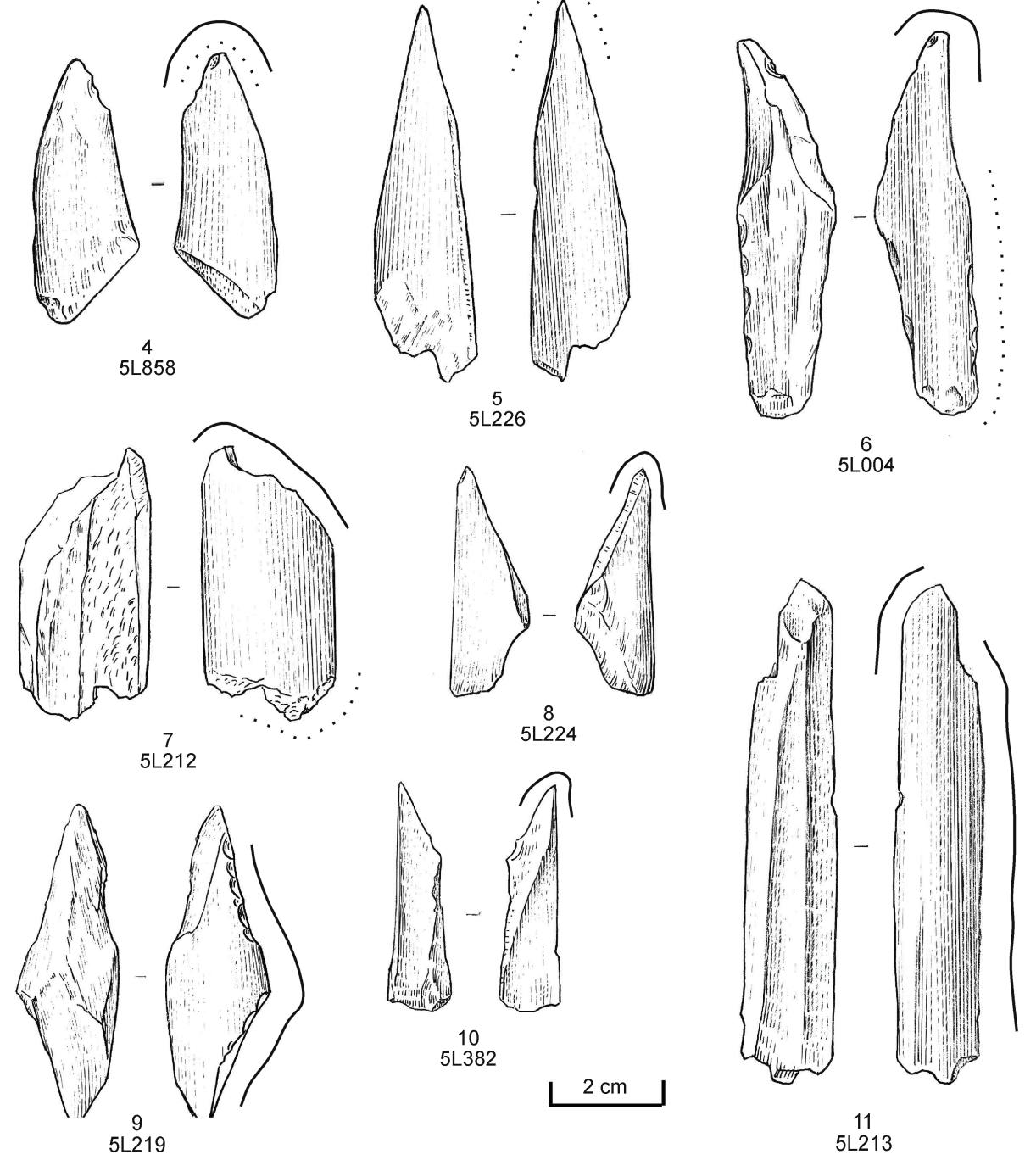

图 3 灵井许昌人遗址部分骨制品微痕观察

标本外实线为使用痕迹部位, 虚线为加工痕迹部位

运动产生的方向性小型片疤, 且在该部位同时出现 钻的功用.

使用光泽, 结合其磨圆特征表明下部尖端可能有过

(iv) 标本 5L212. 该标本的微痕特征较为复杂, 
具有多重类别的微痕, 为较好的对比材料. 具体如下: (1) 尖端的破裂片疤可能是由使用产生的, 至少是由 人为因素造成的, 使用光泽沿工作边缘向下延伸; (2) 上部刃缘带有一系列微小明亮光泽, 为使用痕迹; (3) 右下靠近底部的边缘上有一系列中小型羽状疤, 可 能是人工有意识的加工; (4) 左侧表面有成组平行的 斜向或垂直于长轴边缘的条痕, 比较接近实验标本 EXP001 的加工擦痕, 可认定此处应为石制品刮骨剔 肉的痕迹(图 5); (5) 骨片下端的断裂似人为砍砸的结 果; (6) 背部中部突起部分具有一处动物啃咬痕. 总 之, 该标本主要使用功能区集中在尖端. 据分析, 应 该是作为雕刻较硬物质的工具使用, 以至于产生了 明亮光泽和重度磨圆的微痕.

(V) 标本 5L224. 该标本右侧边缘具有中度磨 圆的痕迹以及连续分布的系列小型疮, 这些特征与 石器捆绑痕迹非常相似 ${ }^{[28]}$. 而且, 由骨骼内面观察 发现有磨擦痕迹, 可能与捆绑有关. 右侧以不均匀分 布的小型羽状疤为特征, 故也具有与左侧相应的类 似捆绑痕迹. 该标本尖端的 4 条脊上都有磨圆, 端口 部重度磨圆. 有 3 条脊在靠近尖端的部位上均有方向 性疤痕, 表明这件标本可能是钻具(图 6). 目前由于 缺乏可对比的实验材料, 该骨器是否被捆绑过作为 复合工具使用, 尚不能确定.

(vi) 标本 5L168. 该标本通过微痕观察, 没有 发现人工打制和使用的痕迹; 背面严重风化, 边缘为 自然断裂.

(vii) 标本 5L213. 该标本的使用痕迹分布在左 侧上部, 有明显使用光泽、重度的磨圆、小型羽状片 疤及少量折断型片疤. 左侧刃缘附近还有少量与之 平行的条痕, 推测这一部位用于切或削片运动. 折断 型片疤是较强作用力的结果, 由此推测可能以骨质 和木质材料为加工对象. 右侧不均匀地分布着若干 尾端为羽状和折断状的小型片疤, 刃缘附近有与之 平行的条痕, 疑似捆绑痕迹. 该骨骼初期加工痕迹表 现在骨表, 有若干组与骨长轴斜交的条痕, 与实验标 本 EXP001 的加工痕迹相当. 自然和生物因素形成的 痕迹包括背部有一组月牙形凹坑, 似为啮齿类动物 的咬痕, 而中部偏下的位置上有裂纹, 应为植物根系 生长时对骨表发生作用的结果.

(viii) 标本 5L004. 该标本右侧有明显的人工二 次加工痕迹. 背面有人工刻划痕迹和动物咬痕. 其尖 部具有中度磨圆痕迹并带有条痕, 可能是作为钻器

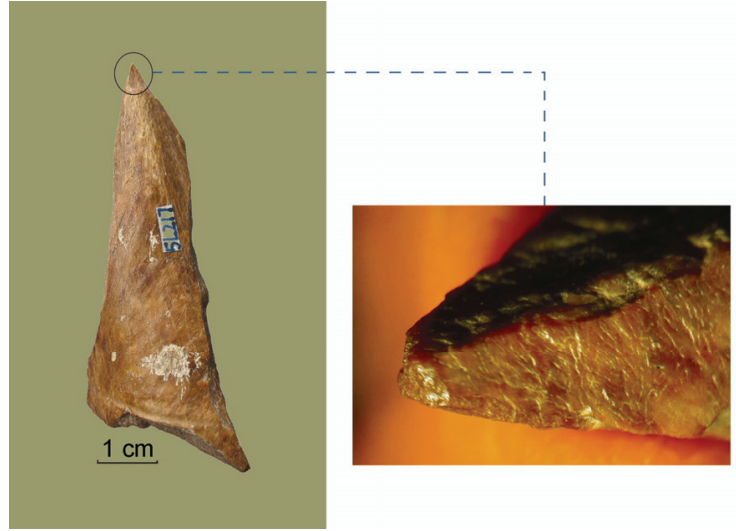

图 4 标本 5L217 尖部使用微痕观察

重度磨圆, 中型破裂片疤, 旋转运动产生的方向性小型片疤及黯淡光泽
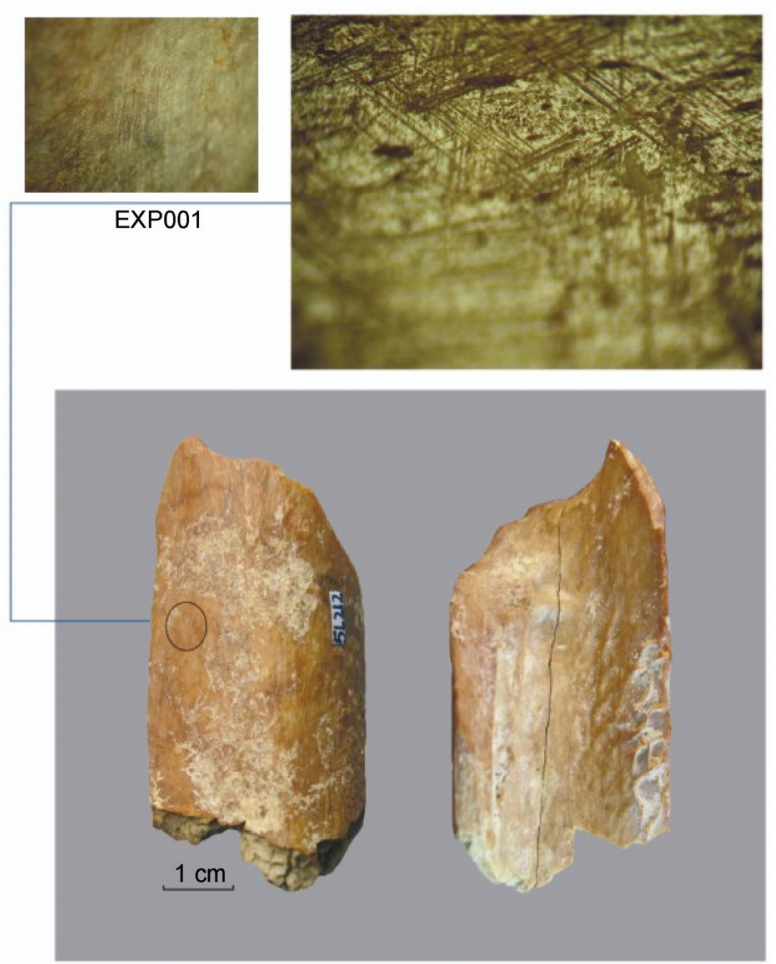

图 5 标本 $5 \mathrm{~L} 212$ 使用微痕观察

骨骼表面可能因由石制工具刮骨清理表面的平行擦痕可与实验标本 EXP001(左上)的刮骨擦痕比较

使用后的结果(图 7).

(ix) 标本 5L382. 该标本尖端是主要的功能区, 证据是重度的磨圆及小型的折断状片疤. 由于使用 强度大, 尖端背面偏右部分出现磨损塌凹.

(X) 标本 5L858. 该标本的尖端由人工修理而 成, 随后经过使用. 尖刀严重磨圆, 并产生使用光泽. 


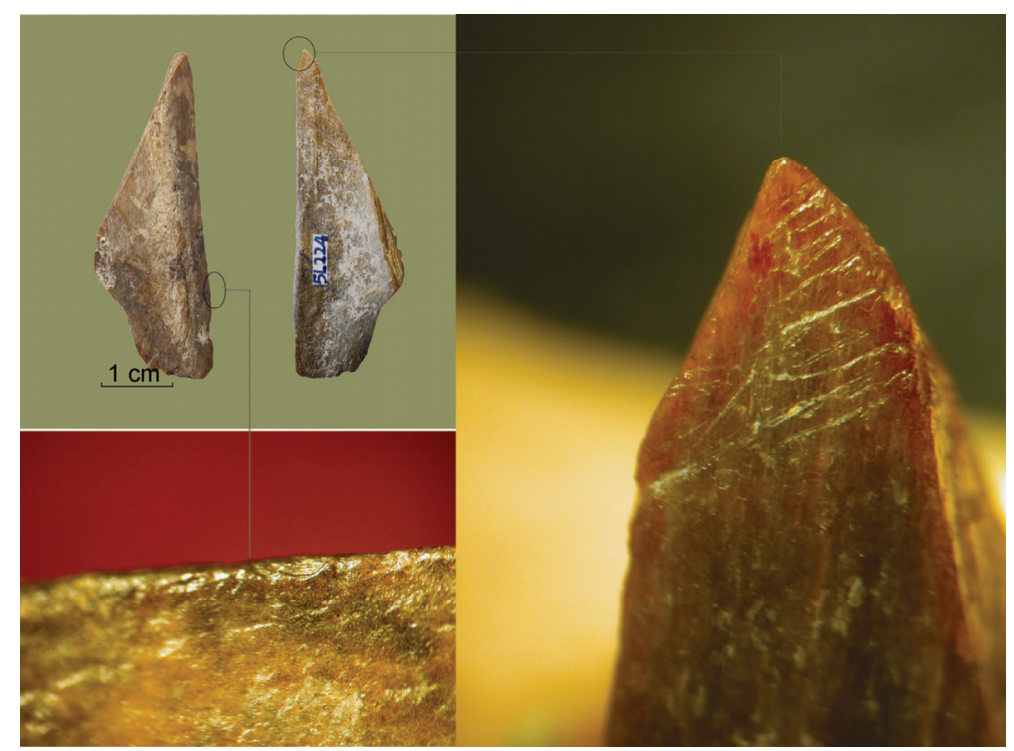

图 6 标本 5L224 使用微痕观察

右图尖部因作为钻器使用后表现出重度磨圆和旋转运动残留下的方向性片疮, 左下图的微痕与石器拥绑实验的微痕极为相似

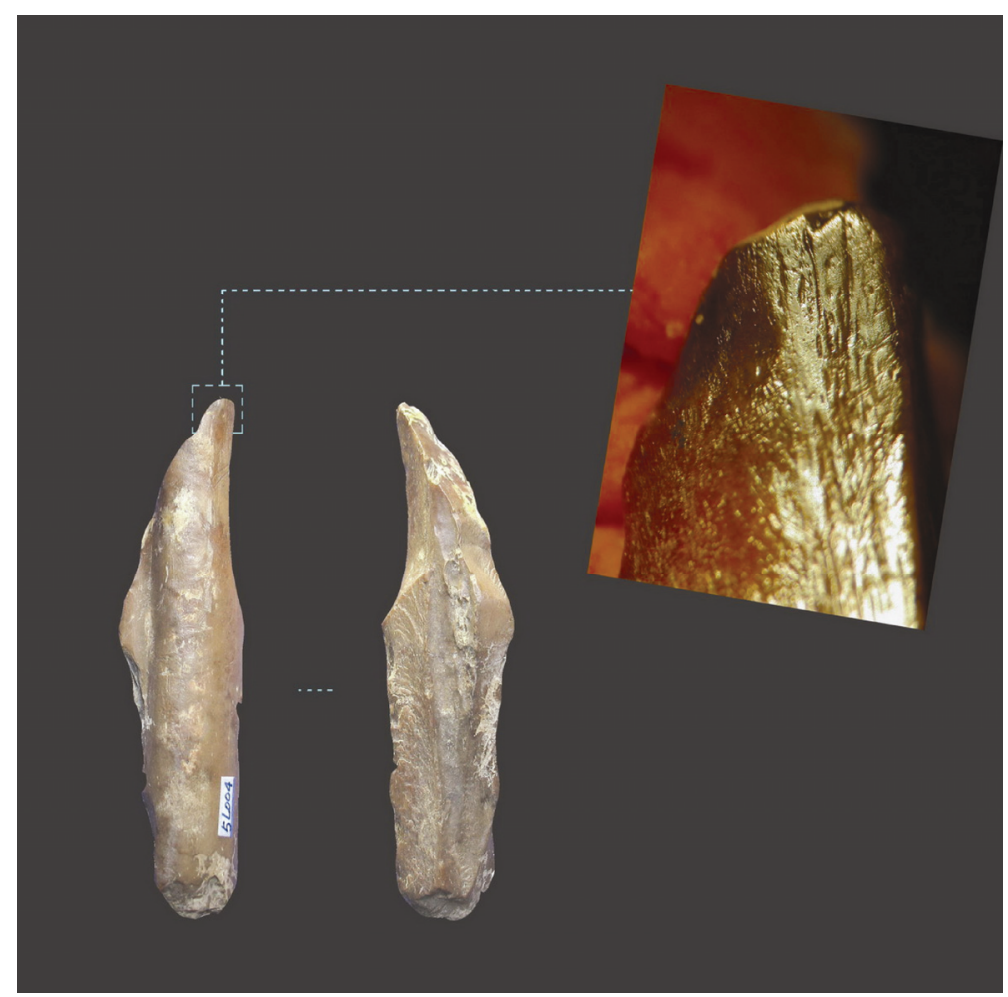

图 7 标本 $5 \mathrm{LO04}$ 使用微痕观察

尖部有作为钻器的人工改造痕迹, 出现重度磨圆, 方向性片疤, 黯淡光泽

尖端的背面有层叠的大型阶状疤，可能是穿刺的结 果. 右侧连续分布小型羽状片疤, 并有垂直于边缘 的短小条痕, 与捆绑痕迹相似. 左侧相对处也有相
似的捆绑痕迹。总之，该标本经修理形成尖端，进 行穿刺活动. 是否经过捆绑成为复合工具, 还需进 一步观察. 


\section{2 痕迹成因和使用功能分析}

通过观察，我们发现该遗址骨制品上痕迹的形 成基本上受到 3 方面因素的影响, 即物理因素(主要 是风化和水流冲刷)、生物因素(啮齿类啃咬和根系生 长作用)和人工作用(制作加工和使用). 基于这些材 料, 我们认为灵井古人类确实有制作与使用骨制品 的行为. 除少数标本外，刃缘一般没有经过刻意加工 修理而是直接利用. 11 件标本中, 8 件具有使用痕迹, 占 $73 \%$; 只有加工痕迹而没有使用痕迹的有 1 件, 占 $9 \%$; 没有人工痕迹的有 2 件, 占 $18 \%$. 在确认经过使 用的骨制品，即骨制工具中， 2 件用于切， 1 件用于刮 削, 2 件用于雕刻, 1 件用于穿刺, 3 件具有钻的功能预 处理(刮磨骨表)，相应的加工对象包含软性、中性和 硬性的各种硬度材料. 使用的 8 件骨制工具中有 3 件 具有疑似捆绑痕迹, 是否是复合工具还需要进一步 观察确定. 另外, 标本 5L217 和 5L213 具有雕刻和钻 两个功能区. 因此我们得到的一个初步结论是: 灵井 许昌人遗址出土的动物骨骼中，有部分标本能基本 确定为有意识的人工制作加工和有目的地使用所产 生的骨制工具.

\section{4 讨论与问题}

早在 20 世纪 30 年代已经有学者开始关注动物骨 骼破碎成因和人工打制骨器的鉴别, 裴文中先生提 出要谨慎对待打制骨器的判定, 必须从实验中观察 动物骨骼破裂的原因和人工痕迹, 并总结出规律性 的东西, 为鉴定遗址中的出土标本提供参考依据. 但 这项研究在后来的很长一段时间里并没有引起广泛 关注和深人探讨，直至 20 世纪 70 年代后期，吕遵谔 等人 ${ }^{[29]}$ 进行了系统的实验，观察不同动物骨骼破碎 的痕迹, 总结出大型食肉类动物啃咬骨骼、人工敲骨 吸髓和人工打击骨片产生的规律性特征。他们的研 究在相当大程度上建立了用肉眼区分和辨别动物行 为产生的痕迹与人为活动产生的痕迹的方法. 龙凤 哼融]曾经对马鞍山出土的 1131 件碎骨表面上的痕迹 作过比较细致全面的研究. 最近, 张乐 ${ }^{[25]}$ 在此基础 上对马鞍山遗址的动物骨骼的痕迹重新进行了观察. 他们对不同的骨骼痕迹作了详尽的分析，包括自然 原因造成的痕迹、处理猎物行为造成的切割痕、敲骨 取髓行为的敲砸疤痕以及烧烤痕迹等. 虽然他们的 分析结果表明在马鞍山遗址中还没有发现有意识
制造和使用的骨制工具, 但他们的研究提示我们在 认识遗址中出土的动物骨骼时绝不能忽视埋藏因素, 并要与人工作用痕迹区分开来. 我们的工作是在上 述工作思路和经验的基础上，从区别动物骨骼上自 然和人工痕迹及人工有意识和无意识加工并使用骨 器的角度, 通过实验和微痕分析方法, 为辨别骨制工 具的制作和使用提供更为充分的证据 ${ }^{[9]}$.

尽管大量的实验观察帮助我们认识了物理与生 物因素在碎骨上形成的各种痕迹，但在过去的动物 骨骼痕迹研究方面很少涉及系统的微痕观察与分析 方法, 也相对缺少对骨器加工和使用方面的探讨. 近 年来对骨器使用微痕的实验和研究在西方学术界受 到密切的关注. 法国学者 Legrand 和 Sidera ${ }^{[22]}$ 对骨雉 使用进行的微痕实验可以为进一步为作为钻器的灵 井骨制工具微痕研究提供可靠的对比分析材料.

当然我们对灵井遗址出土骨制品的研究还是初 步的, 观察的样品较少, 今后需要开展系统的分析. 由于当时研究条件的限制, 我们对灵井骨器的微痕 观察还需要采用高倍的显微镜进一步核实. 因此还 需要在研究方法和视角方面加强探索. 首先, 需要增 加实验，根据古人对骨器和石器多种合理的利用方 式和加工对象, 完善实验设计; 其次, 选择不同部位 的动物骨骼, 设计尽可能完整的骨器加工程序和使 用方法，进行加工、使用方式及功效的模拟实验; 再 次, 还可进行埋藏学方面的实验, 以检验和确认埋藏 因素下形成的微痕特征. 同时, 对遗址中出土的大量 石制工具与骨制工具的制作、类型和使用部位的区别 与联系进行对比，探索骨、石制工具模式之间的相互 依存、功用互补等内在规律.

\section{5 小结}

通过微痕实验和观察, 我们可以鉴别出灵井遗 址出土骨制品上难以用肉眼发现的各种痕迹，确定 人工有意识的加工和使用的痕迹. 由此得到一些初 步但关键的认识：第一，灵井出土骨质遗物痕迹成因 复杂, 在各种埋藏因素和人类活动作用下形成, 因此 痕迹性质不单纯; 第二，出土的骨制品中有一定比例 的骨制工具，使用功能相对多样；第三，使用过的骨 器大多没有经过专门加工, 而是直接使用, 但也有少 量经过二次加工的成形骨器; 第四, 器物功能与形态 之间没有绝对的对应关系，比如有些类型学上分类 为尖状器的骨器在此并没有被使用或者不具备钻或 
穿刺的功能. 微痕分析证实，中国北方晚更新世早期 古人类在河南灵井遗址已经有目的地制作和使用骨
制工具，这一发现证实了骨制工具的使用与石器一 样, 是古人类技术发展和行为文化一个重要方面.

致谢北京大学文博学院的曲殁丽博士参加了骨器微痕实验和观察. 在文章撰写过程中, 与中国科学院古脊椎动物与古 人类研究所的张晓凌博士、皇家安大略博物馆的秦小丽博士、复旦大学的陈虹进行了有益的讨论. 图 3 中的骨制 品线图由河南省文物考古研究所的杨玉华先生绘制。在此一并表示感谢!

\section{参考文献}

1 李占扬. 许昌灵井旧石器时代遗址 2005 年出土石制品初步研究. 人类学学报, 2007, 26: 138-154

2 李占扬, 陈文利. 许昌灵井旧石器时代遗址埋藏学观察. 华夏考古, 2007, 4: 130-136

3 李占扬, 张双权. 灵井遗址新材料及初步研究. 见: 第十一届中国古脊椎动物学学术年会论文集. 北京: 海洋出版社, 2008. 73一83

4 李占扬, 董为. 河南许昌灵井旧石器时代遗址哺乳动物群的性质及时代探讨. 人类学学报, 2007, 26: 345-360

5 李占扬, 张双权. 东亚现代人类起源的重大突破——“河南许昌灵井古人类头盖骨化石专家研讨会”纪要. 中国文物报, 2008 年 2 月 8 日

6 李占扬. 许昌灵井遗址 2006 年发掘报告. 考古学报, 2010, 1: 127-152

7 LeMoine G M. Bone tools and bone technology: A brief history. In: St Pierre C G, Walker R B, eds. Bones as Tools: Current Methods and Interpretations in Worked Bone Studies. Oxford: Archaeopress, 2007. 9-22

8 李超荣, 冯兴无, 郁金城. 王府井东方广场遗址骨制品研究. 人类学学报, 2004, 23: 13-24

9 沈辰, 曲捐丽. 辨别骨质工具的新方法——微痕观察. 中国文物报, 2006 年 7 月 7 日

10 Semenov S A. Prehistoric Technology. London: Cory, Adams and Mackay, 1964

11 Keeley L H. Experimental Determination of Stone Tool Uses. Chicago: The University of Chicago Press, 1980

12 Odell G H. Stone Tools and Mobility in the Illinois Valley: From Hunter-gatherer Camps to Agricultural Villages. Michigan, Ann Arbor: International Monographs in Prehistory, 1996

13 沈辰, 陈淳. 微痕研究(低倍法)的探索与实践——兼谈小长梁遗址石制品的微痕观察. 考古, 2001, 7: 62一73

14 Shen C. The Lithic Production System of the Princess Point Complex during the Transition to Agriculture in Southwestern Ontario, Canada. BAR International Series 991. Oxford: John and Erica Hedgers Ltd, 2001. 45-53

15 St-Pierre C G, Walker R B. Bones as Tools: Current Methods and Interpretations in Worked Bone Studies. Oxford: John and Erica Hedgers Ltd, 2007

16 Newcomer M. Study and replication of bone tools from Ksar Akil (Liban). World Archaeol, 1974, 6: 138_-153

17 Olsen S L. Identification of stone and metal tool marks on bone artifacts. In: Olsen S L, ed. Scanning Electron Microscopy in Archaeology. Oxford: Archaeopress, 1988. 337-360

18 Olsen S L. Introduction: Applications of scanning electron microscopy to archaeology. In: Olsen S L, ed. Scanning Electron Microscopy in Archaeology. Oxford: Archaeopress, 1988. 3-7

19 Runnings A L, Bentley D, Gustafson C E. Use-wear on bone tools: A technique for study under the scanning electron microscope. In: Bonnichsen R, Sorg M, eds. Bone Modification. Orono: Center for the Study of the First Americans, Institute for Quaternary Studies, University of Maine, 1989. 259-266

20 Shipman P, Rose J J. Bone tools: An experimental approach. In: Olsen S L, ed. Scanning Electron Microscopy in Archaeology. Oxford: Archaeopress, 1988. 303-335

21 Campana D V. The manufacture of bone tools in the Zagros and the Levant. MASCA J, 1987, 4: 110-123

22 Legrand A, Sidera I. Methods, means and results when studying European bone industries. In: St Pierre C G, Walker R B, eds. Bones as Tools: Current Methods and Interpretations in Worked Bone Studies. Oxford: Archaeopress, 2007. 67—80

23 St Pierre C G. Bone awls of the St. Lawrence Iroquoians: A microwear analysis. In: St Pierre C G, Walker R B, eds. Bones as Tools: Current Methods and Interpretations in Worked Bone Studies. Oxford: Archaeopress, 2007. 107—118

24 Buc N, Loponte D. Bone tool types and microwear patterns: Some examples from the Pampa Region, South America. In: St Pierre C G, Walker R B, eds. Bones as Tools: Current Methods and Interpretations in Worked Bone Studies. Oxford: Archaeopress, 2007. 143-158

25 张乐. 马鞍山遗址古人类行为的动物考古学研究. 博士学位论文. 北京: 中国科学院古脊椎动物与古人类研究所, 2008

26 张双权. 河南许昌灵井动物群的埋藏学研究. 博士学位论文. 北京: 中国科学院古脊椎动物与古人类研究所, 2009

27 高星, 沈辰. 石器微痕分析的考古学实验研究. 北京: 科学出版社, 2008 
28 赵静芳, 宋艳花, 陈虹, 等. 石器捆绑实验与微痕分析报告. 见: 高星, 沈辰, 编. 石器微痕分析的考古学实验研究. 北京: 科学出 版社, 2008. 145-176

29 吕遵谔, 黄蕴平. 大型食肉类动物啃咬骨骼和敲骨取髓破碎骨片的特征. 见: 北京大学考古系, 编. 纪念北京大学考古系专业三十 周年论文集. 北京: 文物出版社, 1993. 4-39

30 龙凤骧. 马鞍山遗址出土碎骨表面痕迹的分析. 人类学学报, 1992, 11: 217-229

- 动 态 .

\section{忩安动物群胚胎化石三维结构展现出两侧对称动物特征}

来自贵州瓮安埃迪卡拉纪陡山沱组的动物胚胎化石是 目前地球上发现的最古老多细胞动物化石. 其中的很多胚 胎都是早期分裂胚胎, 大部分只能给出有限的系统发生信 号. 中国科学院南京地质古生物研究所陈均远研究小组与 合作者利用同步辐射 X 射线相衬显微 CT 技术, 对新发现 的两颗翁安动物群胚胎化石进行了三维结构重建. 结果显 示这两颗胚胎化石已经开始了细胞的迁移和分化, 并在此 基础上产生了极性, 即出现了前后轴、背腹轴和左右轴. 这些显著生物学特征表明它们与两侧对称动物有着十分密
切的亲缘关系. 值得指出的是, 这两颗肧胎化石在细胞迁 移和重排的过程中采用了完全不同的机制, 暗示两者可能 来自不同的分类群, 研究人员推测它们至少已经分离 4000 万年, 说明两侧对称动物不仅仅在新元古代就已经出现, 而且有了相当程度的分化, 这为了解寒武纪大爆发之前后 生动物的演化历程提供了重要线索. 相关研究论文发表在 2009 年 11 月 20 日 $P N A S, 106(45): 19056$-19060 上.

(信息来源：科学技术部《基础科学研究快报》) 